\title{
Analysis and Research of Structure and Process Characteristic Based on Low Rigidity Shell Part
}

\author{
Liqin Miao*, Hang Yu, Wanrui Cui, Heping Jiang, Yuxin Li, Ruoping Wang and Xiaojiao Gao \\ Department of numerical design \& manufacture \\ Changchun Research Institute of Equipment and Technology, Changchun 130012, P.R.CHINA
}

\begin{abstract}
The process design is a very important step in NC machining which will ensure the manufacturing quality of the part. For the low rigidity part, due to the low rigidity and high deformation under certain load, herein, the low rigidity part often shows more clamping deformation under clamping force and cutting force in manufacturing, so that it will lead to machining error out of tolerance. In this paper, we choose a low rigidity shell part as research target, the machining process will be designed and analyzed, two clamping schemes were designed for this part, and the comparison and analysis were finished by using finite element method (FEM), the results showed that the smaller deformation and error was obtained in the axial clamping scheme, therefore, we choose this scheme as the ultimate clamping scheme for the low rigidity shell part.
\end{abstract}

\section{Introduction}

With the development of science and technology, all kinds of advanced machining technologies were applied on manufacturing, especially, with the development of computer technology, the numerical control(NC) machining technology developed rapidly, so far, it has been main manufacturing technology. It has been an important subject to improve the machining efficiency and reduce the cost. The machining scheme design is a very important step in $\mathrm{NC}$ machining which in general includes reasonable analysis, machining process analysis, process route and machining method making, clamping scheme design, and machining tools choosing. In addition, the optimization of process parameters is also important. Different optimization methods for cutting parameters were used in [1-3]. The optimization of cutting dosages in milling was researched in [4].In order to save energy, the optimization of cutting parameters was finished in [5]. The influence of cutting parameters on cutting temperature in milling was discussed in [6]. The work hardening capability of stainless steel together with its mentioned mechanical and thermal properties results in severe cutting tool wear and low surface quality of the machined surface[7-8]. The concern towards green manufacturing has attracted many manufacturers to implement a Minimum Quantity Lubrication (MQL) with biodegradable lubricant on their manufacturing activities[9]

The low rigidity part often shows more clamping deformation under clamping force and cutting force in manufacturing, and the machining result is difficult to ensure compare with the regular part, therefore, the design of process and clamping scheme is very important for the low rigidity parts. In this paper, we focus on the low rigidity shell part, as shown in Fig. 1, to design the clamping scheme and analyze the machining process, compare and analyze different schemes so that we can obtain the optimized result, then the machining error should be decreased and the machining result and quality should be ensured.

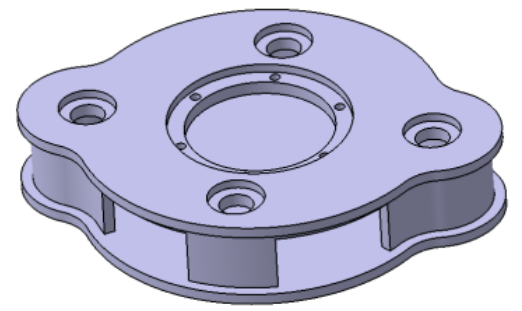

Fig.1 Low rigidity shell part

\section{Clamping force calculaiton}

In this paper, we mainly focus on the top surface and holes manufacturing for the part, to design the machining process. The material of low rigidity shell part is $45 \#$ steel, according to the part material and structure characteristic, the NC milling machine tool and milling method were used for the part.

The formula for cutting force as Eq. 1 .

$$
P=C_{p} t^{0.86} S_{z}{ }^{0.72} D^{-0.86} B z K_{P}
$$

\footnotetext{
a Corresponding author:miaoliqin $@ 163 . c 0 m$
} 
Where $\mathrm{Cp}$ is the coefficient about part material and cutting tool type, it can be referenced in machining handbook; $t$ is cutting $\operatorname{depth}(\mathrm{mm}) ; S_{z}$ is feed per tooth $(\mathrm{mm}) ; D$ is milling tool diameter $(\mathrm{mm}) ; B$ is milling width $(\mathrm{mm}) ; n$ is spindle revolution $\operatorname{speed}(\mathrm{npm}) ; z$ is teeth number; $K_{p}$ is correction coefficient, $K_{p}=\left(\frac{\sigma_{b}}{736}\right)^{0.8} ; \sigma_{b}$ is tensile strength of part material $(\mathrm{MPa})$. The cutting force $\mathrm{P}$ is $110 \mathrm{~N}$ by calculation.

The clamping force includes values, direction, and action point. According to the principle of clamping point choosing, the clamping point should face alignment to the position element, and locate the higher rigidity position and near the machining area of the part. For the clamping force, according to the machining handbook and empirical formula as Eq. 2.

$$
W=K . P
$$

Where $W$ is the actual clamping force $(\mathrm{N}) ; P$ is cutting force(N); $K$ is safety factor, in general, $K=2.5 \sim 3$ for rough machining, $K=1.5 \sim 2.5$ for finishing machining.

Here, $W=200 \mathrm{~N}$.

\section{Radial side push clamping scheme}

In NC machining, when the machining method was made, the fixture was needed to design further to ensure the dimension of machining surface, geometric shape and position precision. The fixture is composed by position element, clamping element, tool direction element, connection element and fixture body. The reasonable fixture can reduce the deformation under load, and ensure machining precision, improve the manufacturing efficiency, reduce the labor intensity, enlarge the machining scope of machine. In this paper, the radial side push clamping scheme was designed first according to the structure characteristic of low rigidity shell part, as shown in Fig. 2(a). In this scheme, the location faces are bottom face and side face of the part, the clamping force acted on the two side faces, screw type clamping unit was used, the $U$ shape slot was made in the fixture body which ensure the connection between machine and fixture. The position error could be decreased due to the application of two side push clamping. The deformation resulted from clamping force was analyzed by using FEM, the analysis results as shown in Fig.2 (b)-(f).

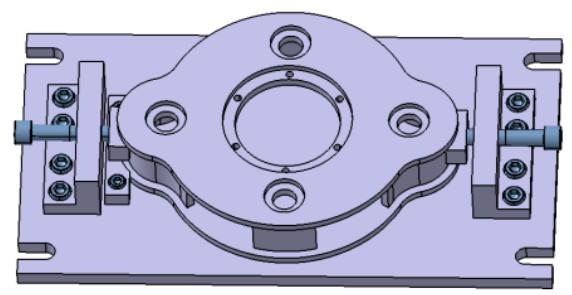

(a) Clamping scheme

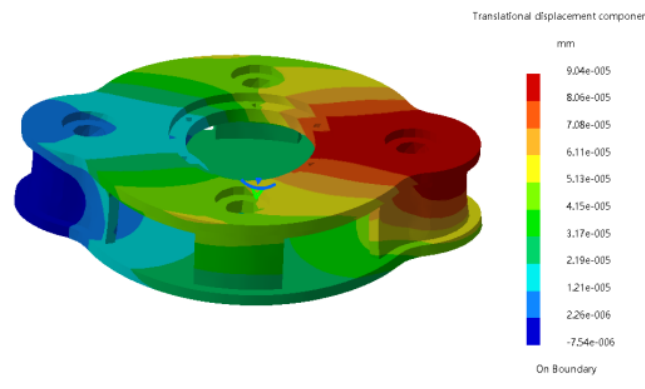

(b) Translational displacement

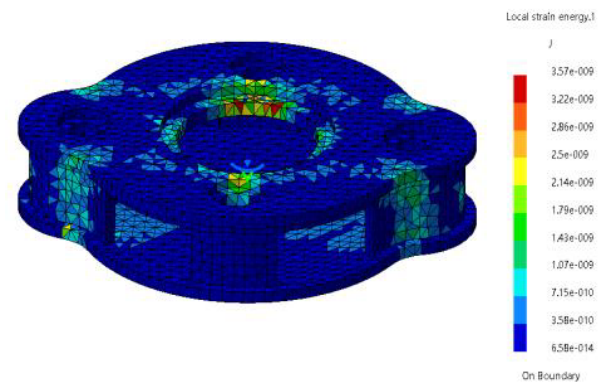

(c) Local strain energy

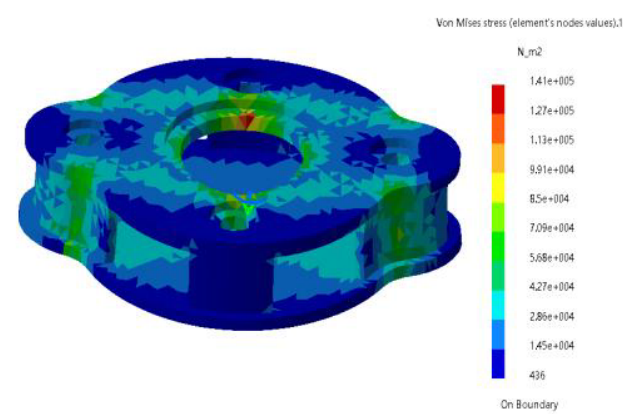

(d) Von Mises stress(element's nodes values)

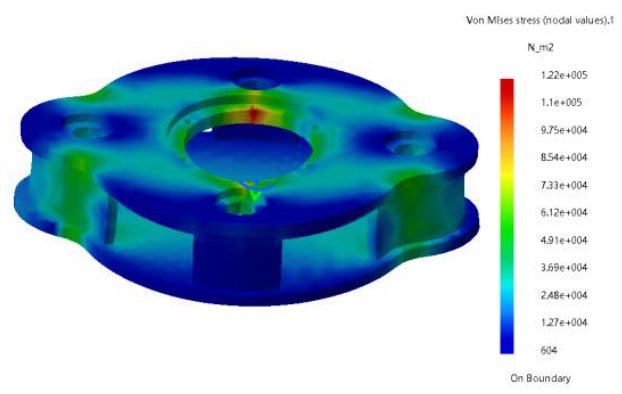

(e) Von Mises stress(nodal values)

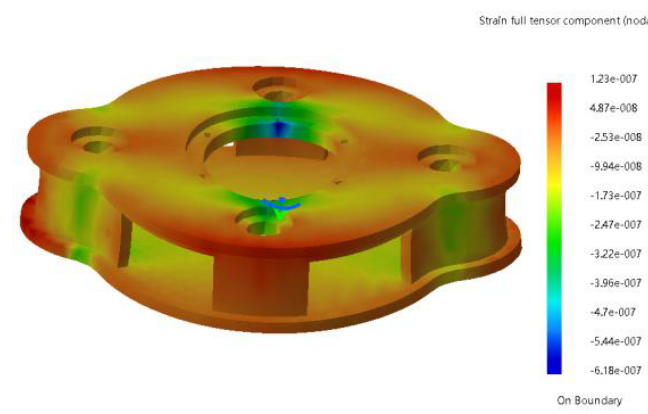


(f) Strain full tensor component(nodal values)

Fig. 2 Analysis results of radial side push clamping scheme

\section{Axial press clamping scheme}

In order to compare and analyze different schemes, the axial press clamping scheme was designed according to the structure of low rigidity shell part, as shown in Fig. 3(a). the location faces are bottom face and side face, but the clamping force action point changed from the side face to the top face, screw type clamping unit was also used. In order to accomplish rapid clamping, a slot was made on the support element for the pressure plate, and a long slot was made on the pressure plate, the pressure plate was supported by coil spring. When load or unload part, loosen the nut, then pressure plate can not fall down to the fixture body because of the support of coil spring. And the pressure plate can slip in the slot of support element, so the clamping efficiency could be improved. The deformation was analyzed further, the results as shown in Fig. 3(b)-(f).

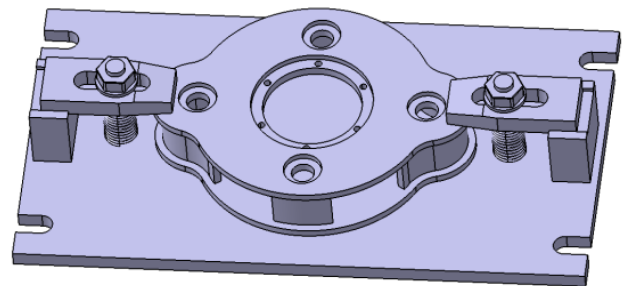

(a) Clamping scheme

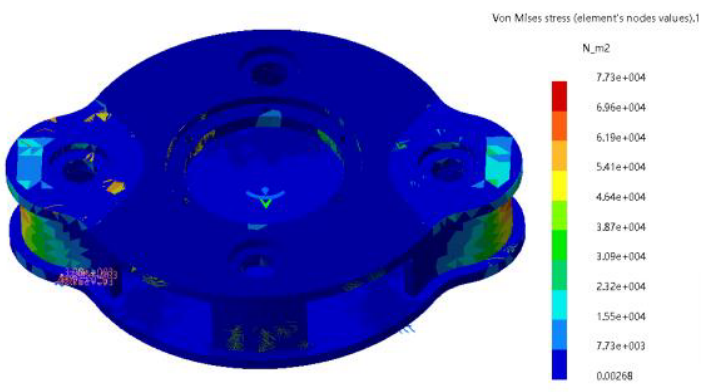

(b) Von Mises stress(element's nodes values)

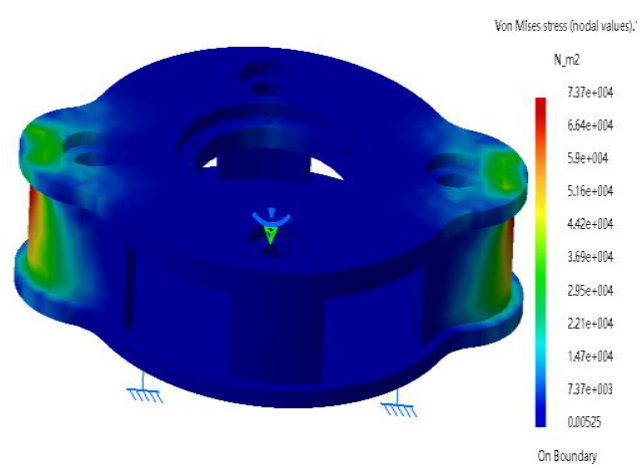

(c) Von Mises stress(nodal values)

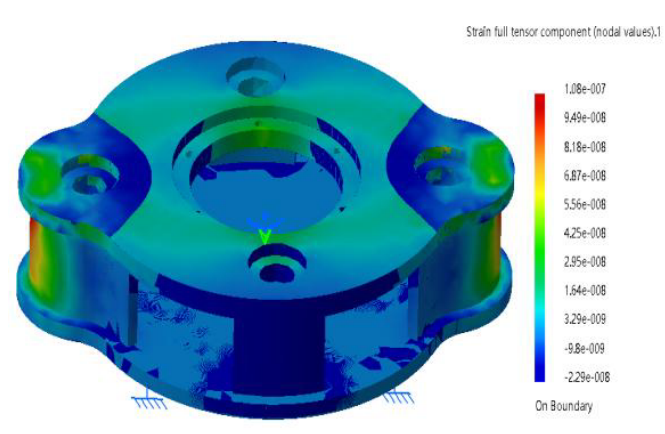

(d) Strain full tensor component(nodal values)

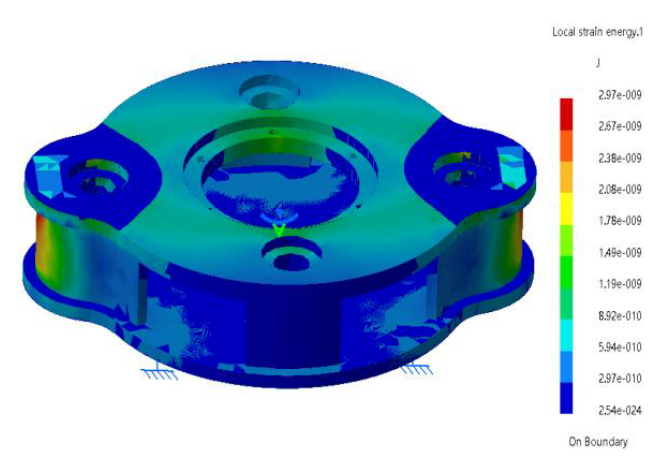

(e) Local strain energy

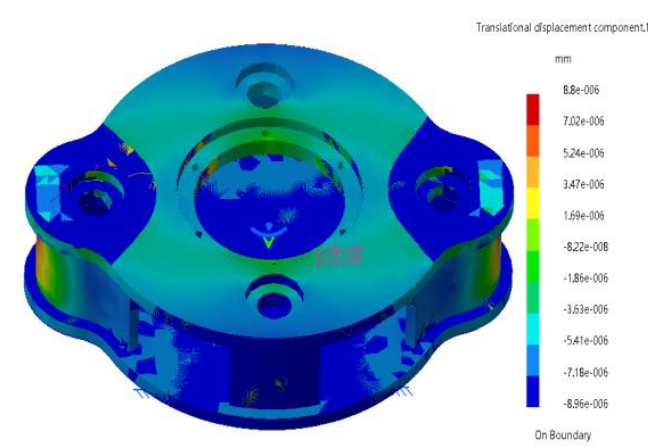

(f) Translational displacement

Fig. 3 Analysis results of axial press clamping scheme

Through the analysis of radial side push clamping scheme and axial press clamping scheme, the results showed that the strain and stress were smaller in axial press clamping scheme, analyzed further, we can conclude that the rigidity of the part is better in axial direction, the connected plate between top and bottom surface has a better rigidity in the axial direction but worse rigidity in the radial direction. Therefore, in the radial side push scheme, the clamping force acted on a low rigidity direction, but acted on a high rigidity direction in axial press clamping scheme, under same clamping force, the strain and stress are smaller in axial press clamping scheme than in radial side push scheme, the machining error results from clamping deformation should decrease, then we choose the axial press clamping scheme as the ultimate scheme for the low rigidity shell part. 


\section{Conclusion}

The machining method was made, and the cutting force and clamping force were calculated by analyzing the structure and machining process of the low rigidity shell part. radial side push clamping scheme and axial press clamping scheme were designed, and the two schemes were compared and analyzed further, results showed that the strain and stress cause by clamping force were smaller in axial press clamping scheme, further discussed we concluded that due to the structure of the shell part, in this scheme, the clamping force acted on the position with better rigidity, clamping force direction coincidence with the higher rigidity direction, herein, the deformation is smaller, then the axial press clamping scheme was used for the low rigidity shell part ultimately.

\section{References}

1. L.X.G, Z.S.R. Research\& Experiment on Optimization of Cutting Elements Based on NC Machining. 2010 3rd International Conference on Advanced Computer Theory and Engineering. 3,179 183. (2010)

2. L.X.G, W.H.W. Optimization Research of Cutting Parameters Based on Orthogonal Experiment Method. Machine Tool \& Hydraulics 39(8),17 19. (2011)

3. Z.S.R, L.X.G. Research on Optimization of CuttingParameters Based on Genetic Algorithm. Applied Mechanics and Materials, 121-126, 4640 4645. (2011)

4. Tao Li, Shiping Chen. The optimization of Cutting dosages in milling. Tool technology,43(2),59 61. (2009)

5. Jian-guang Li, Yong $\mathrm{Lu}$ and Hang Zhao, et al. Optimization of cutting parameters for energy saving. Int J Adv Manuf Technol ,70,117 124. (2014)

6. Yujing Sun, Jie Sun and Jianfeng Li, et al. An experimental investigation of the influence of cutting parameters on cutting temperature in milling Ti6Al4V by applying semi-artificial thermocouple. Int $\mathrm{J} \mathrm{Adv}$ Manuf Technol ,70,765 773. (2014)

7. Korkut, I.; Kasap, M.; Ciftci, I.; Seker, U. Determination of optimum cutting parameters during machining of AISI 304 austenitic stainless steel. // Materials \& Design, 25, 303 305. (2004)

8. Krolczyk, G.; Legutko, S.; Gajek, M. Predicting the surface roughness in the dry machining of duplex stainless steel. // Metalurgija, 52, 259 262. (2013)

9. Izamshah R, Mo J P T\&DingS, Hybrid deflection prediction on machining thin-wall monolithic aerospace components, J Eng Manuf, 226,592 605. (2012) 